\title{
QUESTIONS DE NOMENCLATURE CONCERNANT LE GENRE RAILLIETINA FUHRMANN (SYN.: DAVAINEA BL.)
}

\author{
Par 0. FUHRMANN
}

Dans notre travail: Considérations générales sur les Davainea (1), nous avons cherché à grouper les très nombreuses espèces de ce vaste genre en un certain nombre de genres et de sous-genres, afin de facilitter la détermination très difficile des espèces de ce groupe. Le nom générique de Davainea R. Bl. a été conservé pour les espèces très caractéristiques du type de Davainea proglotina Dav., tandis que la très grande majorité des espèces a été réunie sous le nom de Raillietina Fuhrmann.

Ce vaste genre a été subdivisé en quatre sous-genres : Johnstonia, Skriabinia, Ransomia et Paroniella.

Lors de la création de das sous-genres nous ne nous sommes pas conformés à l'article 9 des Règles internationales de nomenclature, qui veut que lorsqu'un genre est divisé en sous-genres, le nom du sous-genre typique soit le mểme que celui du genre.

De ce fait, un de nos sous-genres doit tomber et recevoir le nom de Raillietina et l'espèce-type de ce sous-genre sera alors en même temps l'espèce-type du genre.

Mais cette question de nomenclature se complique par le fait que Ch. Joyeux, dans un travail récent (2), a trouvé que Raillietina (Johnstonia) echinobothrida (Mégnin, 1881) de la poule, était en réalité une espèce qui normalement présente des pores sexuels unilatéraux, et exceptionneliement, sur une certaine étendue du strobile, des pores sexuels alternes. Raillietina echinobothrida, qui était lie type du sous-genre Johnstonia, rentre ainsi dans le sous-genre Ransomia; mais le premier ayant perdu son type, le nom tombe et doit être remplacé par un autre.

Afin de ne pas produire trop de bouleversement dans la nomenclature du genre Raillietina, nous proposons, d'accord avec le $\mathrm{D}^{\mathrm{r}}$ Joyeux, de remplacer le nom de Johnstonia par celui de Raillietina qui devient ainsi le sous-genre-type du genre Raillietina.

(1) Festschrift für Zschokke, Basel, № 27, 1920.

(2) Joyeux (Ch.). - Recherches sur la faune helminthologique africaine. Arch. Inst. Past. Tunis, XII, fasc. 2, 1923, p. 123.

Annales de Parasitologie, T. II, $\mathrm{N}^{\circ} 4 .-$ Octobre 1924 , p. 312. 
En ce qui concerne l'espèce-type du sous-genre Raillietina, nous proposons comme telle $R$. crassula (Rud. 1819) (1), dont l'espècetype se trouve dans la vaste collection de Rudolphi au Musée de Berlin.

\section{RÉSUMÉ}

Pour les raisons énoncées ci-dessus, notre sous-genre Johnstonia change de nom et de type et devient Raillietina, espèce-type $R$. crassula (Rud. 1819). Notre genre Raillietina comprend donc maintenant les quatre sous-genres suivants : Paroniella, Ransomia, Skriabinia et Raillietina.

Laboratoire de Zoologie de la Faculté des Sciences de Neuchâtel.

(1) R. crassula (Rud.) Clerc, 1906, est une autre espéce appartenant au sousgenre Ransomia à laquelle nous avons donné le nom de Raillietina Clerci Fuhrm., 1920 (Loc. cil.).

Pour la description de l'espèce type de $R$. crassula (Rud. 1819), voir FuhrmanN (O.). Neue Davaineiden. Centralbl. f. Bakt. u. Parasit., Bd. 49, p. 104. 